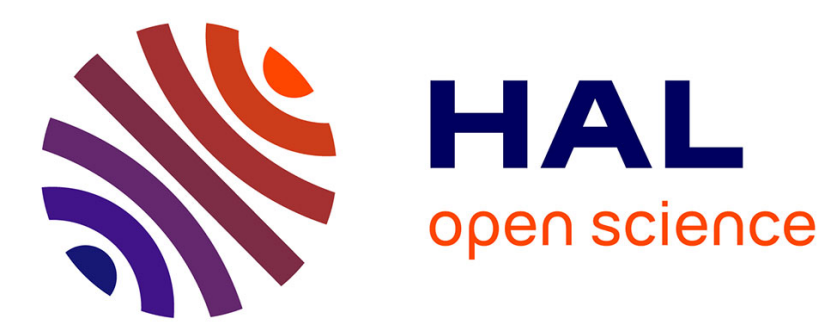

\title{
Fragmentation mechanisms of confined co-flowing capillary threads revealed by active flow focusing
}

Matthieu Robert de Saint Vincent, Jean-Pierre Delville

\section{To cite this version:}

Matthieu Robert de Saint Vincent, Jean-Pierre Delville. Fragmentation mechanisms of confined co-flowing capillary threads revealed by active flow focusing. Physical Review Fluids, 2016, 1 (4), pp.043901 (1-22). 10.1103/PhysRevFluids.1.043901 . hal-01392623

\section{HAL Id: hal-01392623 \\ https://hal.science/hal-01392623}

Submitted on 4 Nov 2016

HAL is a multi-disciplinary open access archive for the deposit and dissemination of scientific research documents, whether they are published or not. The documents may come from teaching and research institutions in France or abroad, or from public or private research centers.
L'archive ouverte pluridisciplinaire HAL, est destinée au dépôt et à la diffusion de documents scientifiques de niveau recherche, publiés ou non, émanant des établissements d'enseignement et de recherche français ou étrangers, des laboratoires publics ou privés.

\section{다(1)(2)}

Distributed under a Creative Commons Attribution - ShareAlikel 4.0 International 


\title{
Fragmentation mechanisms of confined co-flowing capillary threads revealed by active flow focusing
}

\author{
Matthieu Robert de Saint Vincent ${ }^{1,2,3, *}$ and Jean-Pierre Delville ${ }^{2,3, \dagger}$ \\ ${ }^{1}$ IPR, UMR CNRS 6251, Campus Beaulieu, Université Rennes 1, 35042 Rennes, France \\ ${ }^{2}$ Univ. Bordeaux, LOMA, UMR 5798, F-33400 Talence, France \\ ${ }^{3}$ CNRS, LOMA, UMR 5798, F-33400 Talence, France
}

\begin{abstract}
The control over stationary liquid thread fragmentation in confined co-flows is a key issue for the processing and transport of fluids in (micro-) ducts. Confinement indeed strongly enhances the stability of capillary threads, and also induces steric and hydrodynamic feedback effects on diphasic flows. We investigate the thread-to-droplet transition within the confined environment of a microchannel by using optocapillarity, i.e. interface stresses driven by light, as a wall-free constriction to locally flow-focus stable threads in a tunable way, pinch them and force their fragmentation. Above some flow-dependent onset in optical forcing, we observe a dynamic transition alternating between continuous (thread) and fragmented (droplets) states and show a surprising gradual thread-to-droplet transition when increasing the amplitude of the thread constriction. This transition is interpreted as an evolution from a convective to an absolute instability. Depending on the forcing amplitude, we then identify and characterise several stable fragmented regimes of single and multiple droplet periodicity (up to period-8). These droplet regimes build a robust flow-independent bifurcation diagram that eventually closes up, due to the flow confinement, to a monodisperse droplet size, independent of the forcing and close to the most unstable mode expected from the Rayleigh-Plateau instability. This fixed monodispersity can be circumvented by temporally modulating the optocapillary coupling, as we show that fragmentation can then occur either by triggering again the Rayleigh-Plateau instability when the largest excitable wavelength is larger than that of the most unstable mode, or as a pure consequence of a sufficiently strong optocapillary pinching. When properly adjusted, this modulation allows to avoid the transient reforming and multidisperse regimes, and thereby to reversibly produce stable monodisperse droplet trains of controlled size. By actuating local flow-focusing in time and amplitude, optocapillarity thus proves an efficient way to characterise and understand the thread-to-droplet transition in microchannels and to advance channel constriction strategies for the production of tunable monodisperse droplets when the overall confinement is important.
\end{abstract}

\section{INTRODUCTION}

The fractionation of a liquid thread into droplets of controlled size has known a long-standing history and interest [1], dating back from the seminal observations of Savart [2], later rationalised by Plateau [3] and Lord Rayleigh [4-6]. The recent development of digital microfluidics [7] has renewed this interest, and opened new avenues in the formation of bubbles and droplets [8, 9]. Discrete droplets indeed represent individual digits for (bio-)chemical analyses [10] as well as material synthesis [11], motivating the investigation on techniques for dispensing calibrated droplets at high throughput [12]. The main approaches explored to this end relied on the geometrical properties of the channel, for example by focusing a stream of fluid to be dispersed into another immiscible fluid with a lateral constriction [13, 14], or by abruptly releasing a thread confined vertically when reaching a thicker terrace [15-17]. Many active approaches were also prompted to actuate the detachment of individual droplets from a fluid reservoir [18-23], such

\footnotetext{
* rdsv.matthieu@gmail.com

$\dagger$ jean-pierre.delville@u-bordeaux.fr
}

as electric-field induced ejection of droplets from a Taylor cone [24], or a mechanical stimulation of a liquid thread by a transducer $[25,26]$. In the jetting regime, droplet actuation was also provoked by AC electric fields [27], or modulated laser heating of the interface [28].

Fragmentation at low Reynolds number relies on the destabilisation of a liquid thread under local capillary and viscous forces. When the geometrical confinement is important enough to squeeze it, the thread becomes stable in respect with the Rayleigh-Plateau instability $[29,30]$. In addition, non-linear disturbances, such as collective effects due to the traffic in the channel $[31,32]$ or partial clogging from the local inflation of the thread $[33,34]$, affect the local flow conditions and thereby the thread stability. These effects are indeed particularly important in digital microfluidics where the flows are generally confined both vertically and laterally due to the rectangular cross-section of the channels. A rich variety of flow regimes, including jetting [30], periodic [13, 14] or non-periodic [35] dripping was thus reported. Although jetting and dripping regimes are respectively associated with the convective and absolute nature of the capillary instability [36], a unified picture distinguishing the effects of advection, confinement and even droplet traffic feedback on the mechanisms leading to the destabilisation and breakup of a confined thread, and to the sub- 
sequent droplet distribution, is still lacking. In this paper, we explore this issue, by investigating the thread-todroplet transition in strong geometric confinement conditions using optocapillarity, i.e. Marangoni stresses driven by light, as a tunable wall-free flow-focusing constriction [37]. This forcing thus allows to initiate the pinching of an initially stable thread over a wide range in amplitude to unveil the role played by the constriction on the drop generation. We first show surprising transient stages, marked by the alternation of discrete (thread fragmented in trains of droplets) and continuous (thread reformed) flows over time scales of minutes. We interpret these transient stages as a signature of the evolution from a convective to an absolute instability when increasing the thread pinching induced by the optical forcing. We then observe and fully characterise several droplet generation sequences, mono- and multidisperse, that form a bifurcation-reconnection diagram in the drop generation when increasing the forcing amplitude under continuous laser exposure. We show that this reconnection is driven by the confinement which prevents any active control on the droplet size. By temporally chopping the optical excitation, i.e. by modulating both the duration and the initial amplitude of the wall-free constriction, we then evidence a preferred frequency, synchronised with the most unstable Rayleigh mode of the thread, which facilitates the steady fragmentation of the initially stable thread by avoiding both thread reformation and multidisperse droplet regimes. Forced fragmentation within the Rayleigh-Plateau stability domain is also demonstrated in the strong pinching regime. We finally show that, despite the complex feedback operations occurring within confined channels, the modulated forcing of the threadto-droplet transition allows the reversible generation of calibrated droplets of controlled size, provided that the experimental parameters are carefully chosen.

\section{EXPERIMENTAL PROCEDURE}

We force the fragmentation of confined water-in-oil threads into PDMS microfluidic channels [38] using optocapillary pinching. Optocapillarity relies on the generation of tangential stresses on a liquid interface (Marangoni effect) by local laser heating [39, 40]. These stresses induce interface deflections that can lead to the fragmentation of a liquid thread [37].

\section{A. Optocapillary pinching}

The principle of optocapillary pinching of confined threads can be unveiled by a simplified 1D model, detailed in Ref. [37] and briefly summarised below. As sketched in Fig. 1 (a), a water-in-oil thread can be viewed as a superposition of three immiscible liquid layers, of width $H_{2}, 2 H_{1}$ and $H_{2}$ respectively; the density and viscosity of the phase $i=1,2$ are noted $\rho_{i}$ and $\eta_{i}$. We (a)

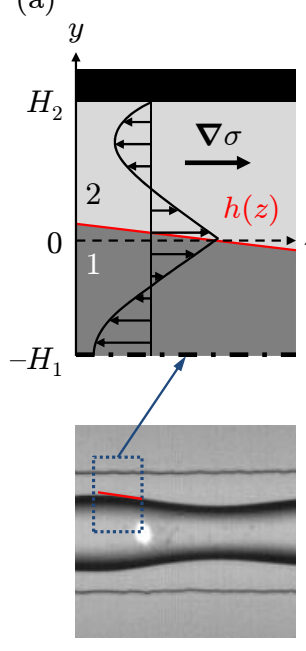

(b)

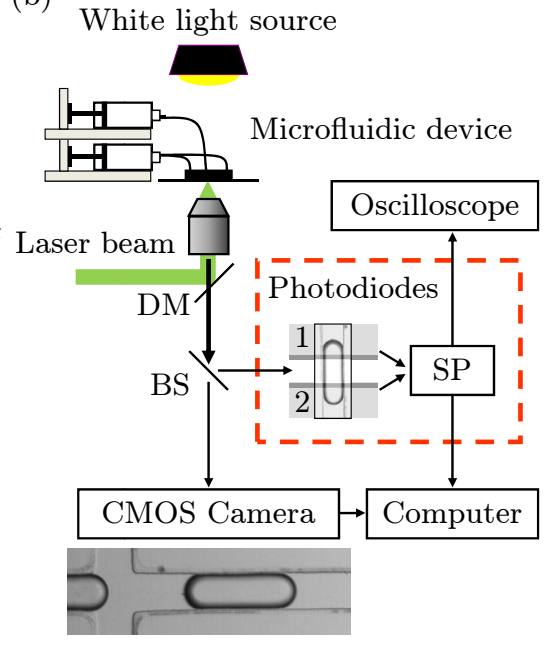

(c)
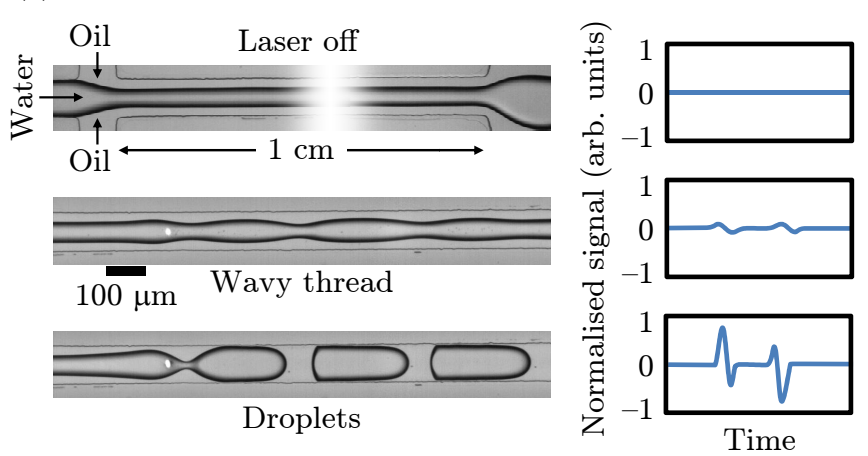

Figure 1. (a) Sketch of the three-layer simplified 1D model of the optocapillary pinching of a confined thread. Only the upper half is represented (dotted box in the experimental image). (b) Sketch of the experimental setup. The same objective is used for both focusing the laser beam and imaging the microchannel onto the detector (dashed box) and a CMOS camera. DM: dichroic mirror; BS: 50/50 beam splitter; SP: signal processing. (c) Left: Typical images of the co-flowing thread. From top to bottom: the unperturbed (laser off), wavy and fragmented cases. The laser spot location is indicated by the white dot due to the dye fluorescence. Right: Corresponding signals as displayed by the oscilloscope when the image of one pattern (wavelength or droplet) passes along a pair of photodiodes connected head-to-tail.

solve the Stokes equation in each phase in the upper half of the system, within the lubrication approximation [velocity field $\left.\boldsymbol{v}_{i}=v_{z, i}(y) \mathbf{e}_{z}\right]$ and assuming incompressible fluids, and set the following boundary conditions: (i) no slip at the wall $\left[v_{z, 2}\left(y=H_{2}\right)=0\right]$, (ii) velocity profile symmetric across $y=-H_{1}\left[\left(\mathrm{~d} v_{z, 1} / \mathrm{d} y\right)_{y=-H_{1}}=0\right]$ and (iii) continuous across the liquid interface $\left[v_{z, 1}(y=0)=\right.$ $\left.v_{z, 2}(y=0)\right]$. In addition, the incompressibility imposes, in a finite system, a deep-layer counterflow as sketched in Fig. 1 (a) that prevents any net flow through each phase $\left[\int_{-H_{1}}^{0} v_{z, 1}(y) \mathrm{d} y=\int_{0}^{H_{2}} v_{z, 2}(y) \mathrm{d} y=0\right]$.

A tangential interfacial tension gradient, $\mathrm{d} \sigma / \mathrm{d} z=$ 
$\eta_{1}\left(\mathrm{~d} v_{z, 1} / \mathrm{d} y\right)_{y=0}-\eta_{2}\left(\mathrm{~d} v_{z, 2} / \mathrm{d} y\right)_{y=0}$, drives interfacial flows in both phases that deform the interface, of profile $h(z)$. For small deformations, solving the Stokes equation with the above assumptions yields

$$
\frac{\mathrm{d} h}{\mathrm{~d} z}=\frac{\ell_{\mathrm{c}}^{2}}{H_{1} H_{2} \sigma_{0}} \frac{\eta_{1} H_{2}^{2}-2 \eta_{2} H_{1}^{2}}{\eta_{1} H_{2}+4 / 3 \eta_{2} H_{1}} \frac{\mathrm{d} \sigma}{\mathrm{d} z},
$$

with $\ell_{\mathrm{c}}=\sqrt{\sigma_{0} /\left(\rho_{1}-\rho_{2}\right) g}$ the capillary length $\left(\rho_{1}>\rho_{2}\right)$. At the point of largest interfacial tension, the deformation should thus be oriented from liquid 1 to liquid 2 if

$$
\frac{H_{2}}{H_{1}} \sqrt{\frac{\eta_{1}}{2 \eta_{2}}}>1
$$

In the presence of surfactants above the critical micelle concentration (CMC), subtle couplings between thermal and molecular transport may occur along the interface, which can be accounted for by an effective coefficient of variation of interfacial tension with temperature, $(\partial \sigma / \partial T)_{\text {eff }}[41]$. We observed this coefficient to be positive in our case $[37,39]$, meaning that the local maximum of interfacial tension coincides with the laser heating spot (neglecting a small shift downstream due to the liquid advection). According to Eq. 2, given the typical layer thicknesses involved on the one hand, and the water and oil viscosities on the other hand, optocapillary stresses thus pinch water-in-oil threads near the laser spot, as represented in Fig. 1 (a). Note that modifying either viscosity or thickness ratios produced opposite behaviours in agreement with Eq. 2 [37].

\section{B. Experimental setup}

We achieve optocapillary forcing by focusing a continuous $\mathrm{TEM}_{00}$ Argon-ion laser (wavelength in vacuum $514.5 \mathrm{~nm}$ ) on the centreline of the flow as illustrated in Fig. 1 (b). A dye, uranine (disodium fluorescein), is added in water $(0.1 \% \mathrm{w} / \mathrm{w})$ to ensure heating occurs by light absorption [39]; photo-degradation and -deposition on the PDMS wall may appear at the beam location after long operating time or at large beam power excitation without modifying the optocapillary coupling thanks to the flow advection. A $\times 5$ microscope objective (Olympus UMPlan Fl, N.A. 0.15) both focuses the beam onto the liquid interface and images the flow in the channel onto a CMOS fast camera (Lightning RTD 16000, 100 frames per second) and a home-made detector sketched in the dashed box of Fig. 1 (b). This detector, described in details in Ref. [42], identifies continuous (thread) and fragmented (droplets) states in real time, and measures the droplet length $L$ in the latter case. Briefly, it detects the variation of illumination on a pair of photodiodes connected head to tail, transversally to the flow, on the passage of a flowing droplet. The projected front and rear interfaces produce a differential signal, corresponding to the contrast in light intensity detected by each photodiode. By knowing the size of the photodiodes and the distance between them, this signal can be processed to calculate the droplet velocity and length. This detector was used to perform continuous measurements over periods of time of several minutes to hours, representing up to several $10^{4}$ individual droplets. Such amount of data would make image analysis cumbersome, assuming image recording is even possible at high rate over such long time.

Water $\left(\eta_{\mathrm{w}}=1 \mathrm{mPas}\right)$ and hexadecane $\left(\eta_{\mathrm{o}}=\right.$ $3.3 \mathrm{mPas})$ are injected at constant flow rates $\left(Q_{\mathrm{w}}\right.$ and $Q_{\mathrm{o}}$, respectively; typical flow velocities are in the $\mathrm{cm} \mathrm{s}^{-1}$ range), meet at a cross-shaped junction, and flow coaxially further downstream as shown on the top image of Fig. 1 (c); when turned on, the laser intercepts the water thread $0.8 \mathrm{~mm}$ downstream of the junction. The rectangular cross-section of the channel (width $w=100 \mu \mathrm{m}$, height $h=55 \mu \mathrm{m}$ ) vertically confines the thread which thereby stabilises it [30]. The flow rate ratio was kept constant, $Q_{\mathrm{w}} / Q_{\mathrm{o}}=2$, so as to ensure similar confinement conditions when varying the thread velocity. To favour thread formation at low flow rates, the interfacial tension is reduced by adding two surfactants, sorbitan monooleate (Span 80, $0.3 \% \mathrm{w} / \mathrm{w}$ ) in oil and sodium dodecyl sulfate (SDS, $2.5 \% \mathrm{w} / \mathrm{w}$ ) in water, both above the CMC. These two surfactants are widely used in digital microfluidic applications; the resulting interfacial tension $\sigma_{0}$ was estimated in the order of $10^{-4} \mathrm{~N} \mathrm{~m}^{-1}$ [43]. Appropriate choices of flow rates produce stationary water-inoil threads, stable during minutes to hours over the whole length of the channel (1 cm, see Fig. 1); the weak vertical squeezing allows to assume a circular cross-section of diameter close to the channel height, $2 R_{0} \simeq h=55 \mu \mathrm{m}$.

Since a stable thread is uniform along the flow direction, the differential signal produced by the detector is constantly zero [top row on Fig. 1(c)]. Turning the laser on at moderate power locally heats the interface, produces Marangoni stresses that deflect the interface and thereby waves the thread, yet not enough to induce its fragmentation [37]. This deformation propagates downstream, leading to subsequent modulations that decay within three to four wavelengths (wavelength $\lambda \simeq 300 \mu \mathrm{m})$ due to the stabilising effect of the confinement. The passage of a node over the photodiodes produces a weak differential signal [Fig. 1 (c), middle row]. Since this signal is much weaker than the one produced by the rear and front interfaces of a droplet, it is interpreted as null and nothing is measured. Increasing the laser power may force the fragmentation of the thread, downstream of the laser spot, into successive droplets; turning the laser off allows the thread to eventually reform. The well defined front and rear interfaces of the droplets thus produce a strong antisymmetric differential signal [Fig. 1 (c), bottom row]. Droplets are individually identified and their passing time, velocity and length are thus individually measured in real time. Individual droplet measurements, collected into a text file, were then processed with a home-written MATLAB ${ }^{\circledR}$ routine to distinguish specific droplet emission sequences and 
Video 1. Movie illustrating the transient fragmentation and reformation of the thread in the continuous (thread) regime. The real-time dynamics is ten times faster; $P=24 \mathrm{~mW}$, $\left(Q_{\mathrm{w}}, Q_{\mathrm{o}}\right)=(2,1) \mu \mathrm{L} \mathrm{min}^{-1}$.

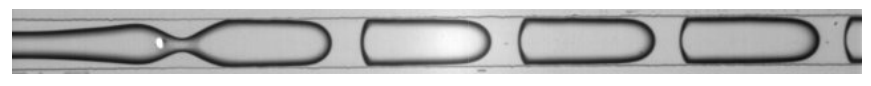

Video 2. Movie illustrating the permanent fragmentation of the thread in the stationary fragmented regime. The thread interface never touches the lateral walls. The realtime dynamics is ten times faster; $P=37 \mathrm{~mW},\left(Q_{\mathrm{w}}, Q_{\mathrm{o}}\right)=$ $(2,1) \mu \mathrm{L} \min ^{-1}$.

continuous thread flow.

This setup therefore allows to make an immediate distinction between continuous (steady or wavy thread) and fragmented (train of droplets) states, and thereby to study the dynamic thread-to-droplet transition.

\section{THREAD-TO-DROPLETS TRANSITION}

To break the thread stability and force its fragmentation, the amplitude of the optocapillary pinching must be sufficient to make the thread locally three-dimensional $[37,44]$. At first sight, this condition seems equivalent to an onset in optocapillary deformation; the thread-todroplet transition should therefore occur abruptly above a threshold laser power. Surprisingly, however, we found this transition not so abrupt, featuring remarkable alternations of continuous and fragmented regimes over a wide power range.

\section{A. Continuous and fragmented states}

The thread-droplet alternation is characterised by recording the passing droplets over long time, under constant laser forcing. Three examples are illustrated in Fig. 2 (a) for $\left(Q_{\mathrm{w}}, Q_{\mathrm{o}}\right)=(2,1) \mu \mathrm{L} \mathrm{min}{ }^{-1}$. Low-power forcing $(P=21 \mathrm{~mW}$, top) does not break the thread immediately; the transient fragmentation of the thread may occur after a few seconds. During this transient stage, the thread first breaks downstream of the laser spot, then it gradually thickens upstream, thus producing droplets of size rapidly increasing from 200 to $500 \mu \mathrm{m}$ (see also Video 1). The thread eventually reforms spontaneously. This process may repeat after arbitrarily long time. A quasi-steady fragmented state, characterised by the regular production of droplets over significant time, can be observed when moderately increasing the laser power ( $P=28 \mathrm{~mW}$, middle). However, in this case the thread ends up reforming after few dozens of seconds. A higher laser power ( $P=37 \mathrm{~mW}$, bottom) is required to finally get a stable fragmented state, producing trains of droplets of uniform length $L \simeq 280 \mu \mathrm{m}$ (Video 2).

The transition from continuous to fragmented states was then characterised by measuring the proportion of liquid dispensed under fragmented state, which in average corresponds to the relative cumulated time interval over which droplets are detected, for a given laser power. Besides, since hysteretic thread-to-droplet transitions were reported in the literature [45], we studied this transition for both increasing and decreasing laser powers. For each measurement, the laser was thus turned on at very low (resp. very high) power, ensuring a continuous (resp. fragmented) initial state, and then gradually increased up (resp. reduced down) to the studied power. Figure 2 (b) depicts this transition for three couples of flow rates, in the increasing (filled symbols) and decreasing power (open symbols) cases. The proportion of droplets gradually increases, from a power threshold and over a power range that linearly shifts up with the imposed flow rates: $17-33 \mathrm{~mW}$ at $\left(Q_{\mathrm{w}}, Q_{\mathrm{o}}\right)=(2,1) \mu \mathrm{L} \mathrm{min}{ }^{-1}, 27-54 \mathrm{~mW}$ at $(3,1.5) \mu \mathrm{L} \mathrm{min}{ }^{-1}$, and $34-74 \mathrm{~mW}$ at $(4,2) \mu \mathrm{L} \mathrm{min}{ }^{-1}$. No significant hysteresis can be noted. The increasing proportion of droplets is associated either to more frequent transient fragmentation-reformation cycles, or to the establishment of longer quasi-steady regimes. The latter are favoured near the upper limit of the transition, and at some particular power values within the transition, leading to remarkable peaks such as around $22 \mathrm{~mW}$ (lowest flow rates) or, to a lesser extent, around $35 \mathrm{~mW}$ (intermediate flow rates).

The flow rate dependence of the transition can be enlighten by reducing the experimental variables to the ratio of the advective to optocapillary charateristic times. As detailed in Ref. [37], one can define an optocapillary time $\tau_{\text {opt }}$ from the tangential stress condition at the interface of the thread, which characterises the dynamics of deformation,

$$
\tau_{\mathrm{opt}}=\frac{\eta_{\mathrm{o}} \omega_{\mathrm{th}} R_{0}}{\varepsilon \Delta \sigma}
$$

where $\omega_{\text {th }} \simeq 10 \mu \mathrm{m}$ is the thermal length scale associated with the laser heating distribution [39], $\varepsilon \sim 0.3 \mu \mathrm{m}$ is the thickness of the lubrication oil films, set by the disjoining pressure [46], and $\Delta \sigma=(\partial \sigma / \partial T)_{\text {eff }} \Delta T$ is the interfacial tension increase with temperature. Assuming $(\partial \sigma / \partial T)_{\text {eff }}$ in the order of $10^{-5} \mathrm{~N} \mathrm{~m}^{-1} \mathrm{~K}^{-1}$ [37], and a local temperature increase of about $1 \mathrm{~K} \mathrm{~mW}^{-1}$ near the laser spot [39], the interfacial tension gradients produced over the thermal length are typically in the range of few $10^{1} \mathrm{~N} \mathrm{~m}^{-2}$ in our experiments. The dependence in beam power $P$ is thus the experimental signature of the physical dependence in interfacial tension gradient.

In presence of flow, $\tau_{\text {opt }}$ should be compared to the advection time,

$$
\tau_{\mathrm{adv}}=\frac{R_{0}}{v_{\|}},
$$

with $v_{\|}$the interface velocity, measured as detailed in Appendix A. The continuous (resp. fragmented) regime is 

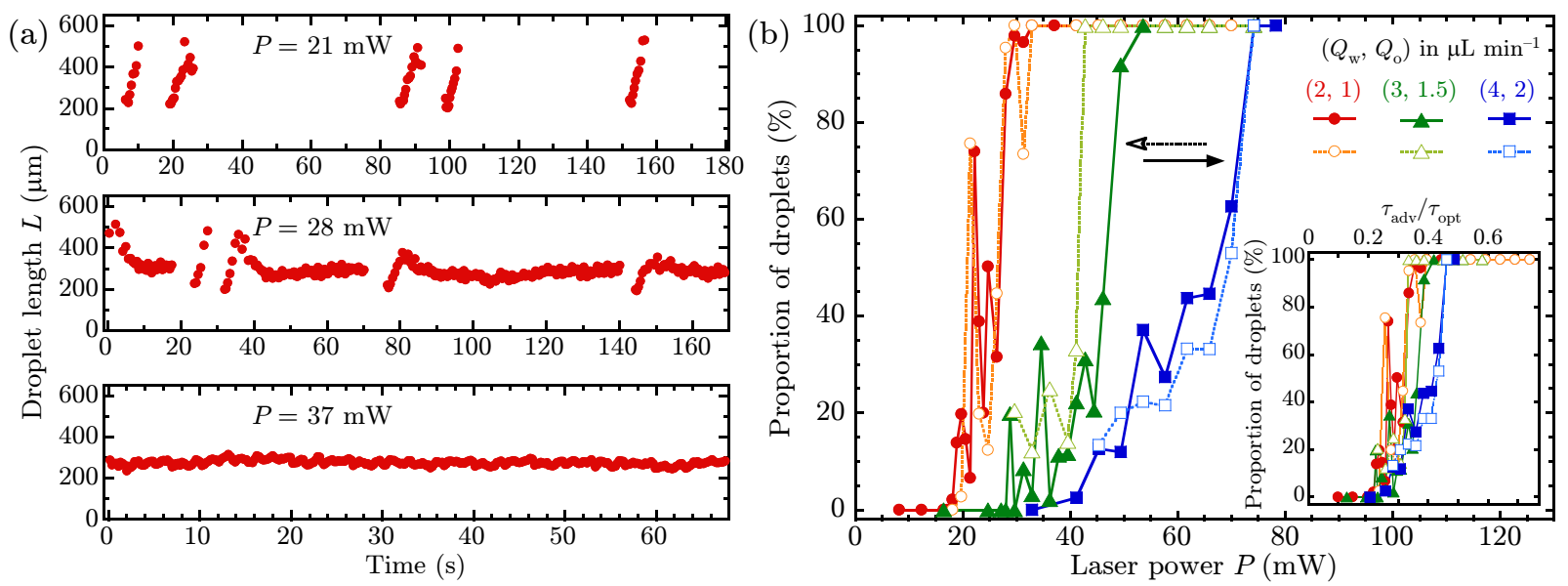

Figure 2. (a) Successive fragmentations and reformations of a microfluidic thread under continuous laser exposure; $\left(Q_{\mathrm{w}}, Q_{\mathrm{o}}\right)=$ $(2,1) \mu \mathrm{L} \mathrm{min}^{-1}$. At $P=21 \mathrm{~mW}$ (top), the flow is essentially continuous (thread), though transient fragmentations punctually occur. At $P=28 \mathrm{~mW}$ (middle), fragmented (quasi-stationary regime) and continuous states coexist. Finally, at $P=37 \mathrm{~mW}$ (bottom) the flow is permanently fragmented (no reformation). (b) Evolution of the proportion of fragmented fluid with laser power (solid-line-connected filled symbols: increasing power, dotted-line-connected open symbols: decreasing power), for the three couples of flow rates investigated. Inset: collapse of the same data when replotted against $\tau_{\text {adv }} / \tau_{\text {opt }}$.

expected for small (resp. large) $\tau_{\text {adv }} / \tau_{\text {opt }}$, with a value at the transition in the order of unity. As illustrated in the inset of Fig. 2 (b), the transition curves nicely collapse when replotted against $\tau_{\text {adv }} / \tau_{\text {opt }} \propto P / v_{\|}$, confirming the linear flow rate dependence of the thread-to-droplet transition as $v_{\|} \propto Q$. Considering the uncertainty on the estimate of $\Delta \sigma$, the inset of Fig. 2 (b) also shows that the thread-to-droplet transition consistently occurs in the expected range in $\tau_{\text {adv }} / \tau_{\text {opt }}$.

\section{B. From convective to absolute instability}

The progressive fragmentation likely results from the establishment of a capillary (Rayleigh-Plateau, RP) instability on the initially stable thread. Such instability would emerge assuming the thread locally loses its vertical confinement over a given length downstream of the laser beam. More specifically, without any forcing the horizontal walls slightly squeeze the thread, which means that the liquid interface is separated from these walls by thin films (of thickness $\varepsilon$ ) due to the total wetting of the oil phase. The laser forcing locally forms a neck that lifts the interface by up to several microns. This neck makes the thread locally unconfined which allows the development of RP instability. The extent of this unstable zone can be estimated by measuring the evolution of the minimal thread radius as it advects within the channel. Figure 3 depicts this evolution for a series of pinching events taken from Video 1. In unfragmented cases (open symbols, dotted lines), the pinching amplifies over typically $300 \mu \mathrm{m}$ (meanwhile the next pinching begins), and then starts to decay. This attenuation confirms that the thread remains stable if neck breakup does not occur when advected over about $300 \mu \mathrm{m}$ (unstable zone for the given couple of flow rates), preventing its fragmentation as observed in the lowest-power region of Fig. 2 (b). However, due to fluctuations of the system, or under stronger forcing, the thread pinching may amplify faster within the unstable zone. The resulting breakup opens a succession of fragmentation events, the first two of which are represented in Fig. 3 (filled symbols, solid lines). In each case, the thread breaks at a distance $L_{\mathrm{b}}$ from the laser spot, below about $300 \mu \mathrm{m}$ in the present case. Its typical evolution during a full series of breakups is represented in Fig. 4 in the transient regime $[P=24 \mathrm{~mW}$, analogous to Fig. 2 (a), top graph]; the images $\mathrm{i}-\mathrm{vi}$ are snapshots at time $t_{k}$, corresponding to the $k$-th breakup. Starting around $330 \mu \mathrm{m}$, the breakup length rapidly decreases, down to a minimum value close to $60 \mu \mathrm{m}$ after about 25 breakups (image iii, $k=27$ ). The breakup length then gradually increases, as the thread thickens, and longer droplets are produced again (image iv, $k=68$ ). Finally, a delayed breakup induces a dramatic increase of $L_{\mathrm{b}}$, as for $k=99$ (image $\mathrm{v}$; note that the next pinching has already started while the breakup occurs). After this last breakup, which occurs slightly below $300 \mu \mathrm{m}$, the next pinching will not amplify enough to break the thread one more time, and eventually decays when reaching the downstream end of the unstable zone (image vi; the droplet emitted at $t_{99}$ is the cropped one at the right edge of the frame). The thread thus reforms.

In contrast, the stationary fragmentation regime features much less variations in breakup length. As also represented in Fig. 4, far beyond the transition $(P=$ $66 \mathrm{~mW}$ ) the breakup length is essentially flat and close to $50 \mu \mathrm{m}$. Variations measured from droplet to droplet actually result from the acquisition rate (100 fps), too slow to accurately resolve each individual breakup event 


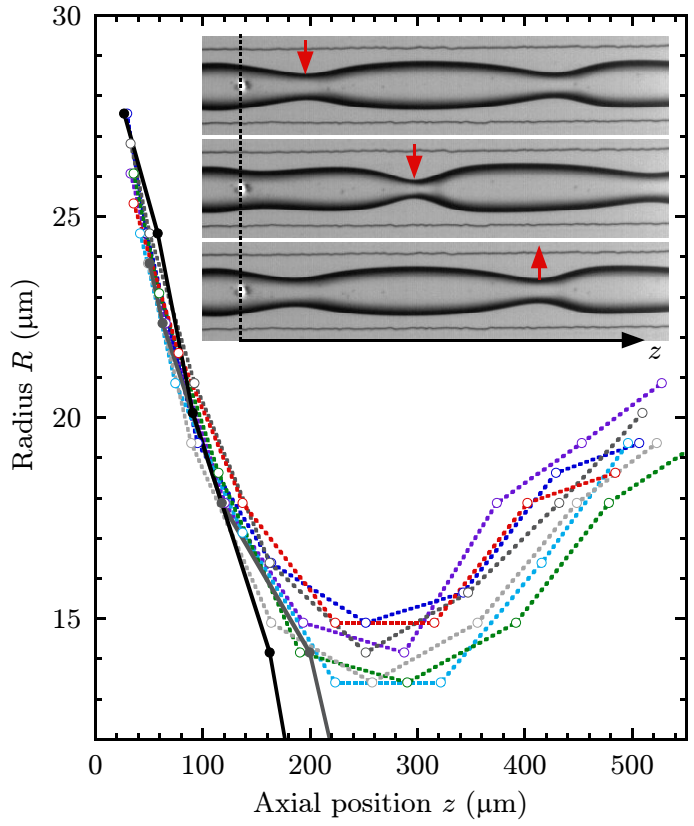

Figure 3. Evolution of the minimal thread radius $R$ along the propagation axis $z$ (origin at the laser spot) of seven distinct pinching events not leading to the fragmentation (open symbols, dotted lines), and the two first breakup events under the same experimental conditions (filled symbols, solid lines). The red, downwards and upwards vertical arrows highlight a specific neck pinching, successively amplifying and decaying. Laser power $P=24 \mathrm{~mW}$, flow rates $\left(Q_{\mathrm{w}}, Q_{\mathrm{o}}\right)=$ $(2,1) \mu \mathrm{L} \min ^{-1}$.

since the ultimate stages of the pinch-off typically scale in the sub-millisecond range [43].

Since the breakup length can equivalently be seen as the persistence length of the unstable thread [47], we can assume that the continuous-to-fragmented state transition corresponds to the gradual evolution from a convective to an absolute instability, or equivalently, to the transition from the so-called jetting to the dripping regime [36]. The instability indeed becomes absolute when an unstable mode exists that nullifies its group velocity at a specific location of the interface in the laboratory frame of reference. In other words, the upstream (hence negative) velocity of the wave packet of unstable perturbations, $v^{*}$, should balance the downstream velocity of the interface $v_{\|}[47]$. When $-v^{*}<v_{\|}$, the instability is convected and breakup occurs far from the laser beam. Conversely, if $-v^{*}>v_{\|}$, the thread always breaks at the upstream end of the unstable region, meaning that its leading edge is located about one radius of horizontal curvature from the laser beam, viz., in this case, $L_{\mathrm{b}} \simeq w / 2=50 \mu \mathrm{m}$ (dripping regime).

The evolution of the persistence length can actually shed light on this transition, as $L_{\mathrm{b}}(k)$ features a 'stroboscopic' evolution of the group velocity during a succession of breakup events. More specifically, an increasing breakup length means that the wave packet

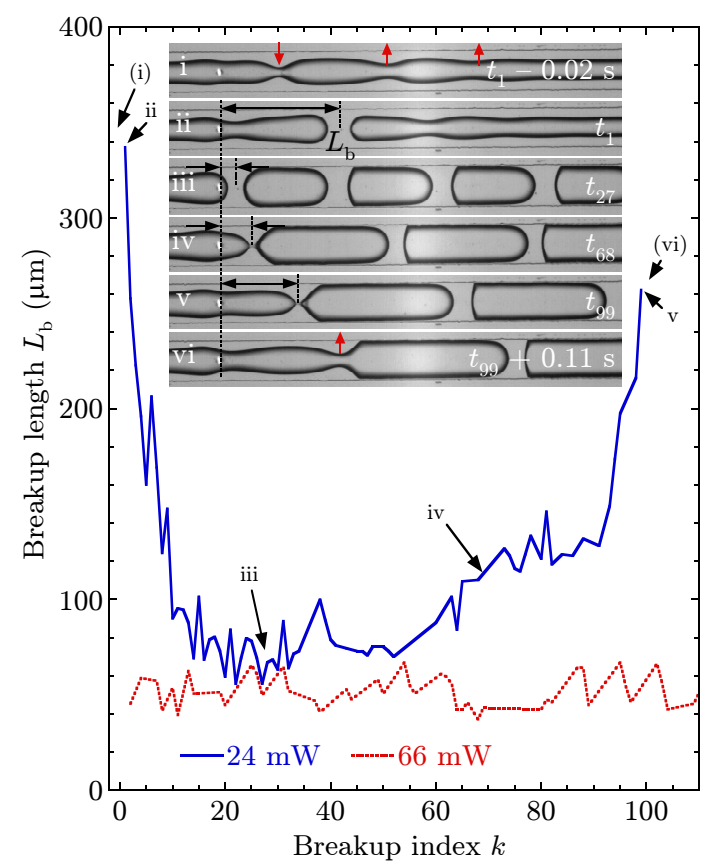

Figure 4. Evolution of the breakup length $L_{\mathrm{b}}$ in a succession of breakup events, for two distinct laser powers, in the transient fragmentation regime [24 $\mathrm{mW}$, analogous to Fig. 2 (a), top], and in the stationary regime $[66 \mathrm{~mW}$, as in Fig. 2 (a), bottom]. Images $\mathrm{i}-\mathrm{vi}$ refer to the transient case represented $(24 \mathrm{~mW})$, for several distinct breakup events $k$ at the corresponding instants $t_{k}$. The red, downwards and upwards vertical arrows depict amplifying and decaying pinchings, respectively. In all cases, $\left(Q_{\mathrm{w}}, Q_{\mathrm{o}}\right)=(2,1) \mu \mathrm{L} \mathrm{min}^{-1}$.

becomes more convected from one breakup to another, and therefore gradually decelerates (in other words, $-v^{*}$ increases). Conversely, a decreasing $L_{\mathrm{b}}(k)$ trend corresponds to a wave packet accelerating backwards; the absolute instability regime should be reached when the breakup length stabilises close to the limiting value $L_{\mathrm{b}} \simeq$ $50 \mu \mathrm{m}$.

Figure 5 compares the evolution trends of the persistence length for four laser powers below (24 and $28 \mathrm{~mW})$, at $(33 \mathrm{~mW})$ and well beyond $(66 \mathrm{~mW})$ the transientlyto-permanently fragmented transition at the considered

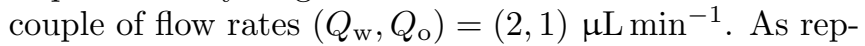
resented in the inset, the $L_{\mathrm{b}}(k)$ curves slowly increase at all powers, yet the trend clearly flattens with increasing power. Note that, in the $24-\mathrm{mW}$ case, only the breakup events in the $k \in[27,93]$ range are considered; the thread dynamics outside this interval is discussed in Appendix B. On the one hand, the progression rate of the thread persistence length, $\mathrm{d} L_{\mathrm{b}} / \mathrm{d} k$, plotted against beam power in Fig. 5, abruptly vanishes to zero below $33 \mathrm{~mW}$, meaning that the instability is convective in the transiently and quasi-stationary fragmented regimes [Fig. 2 (a), top and middle]. On the other hand, above $33 \mathrm{~mW}$ the stabilised breakup length is close to $50 \mu \mathrm{m}$, which confirms that the instability has become absolute. The thread yet reaches a stationary dripping regime and no longer spon- 


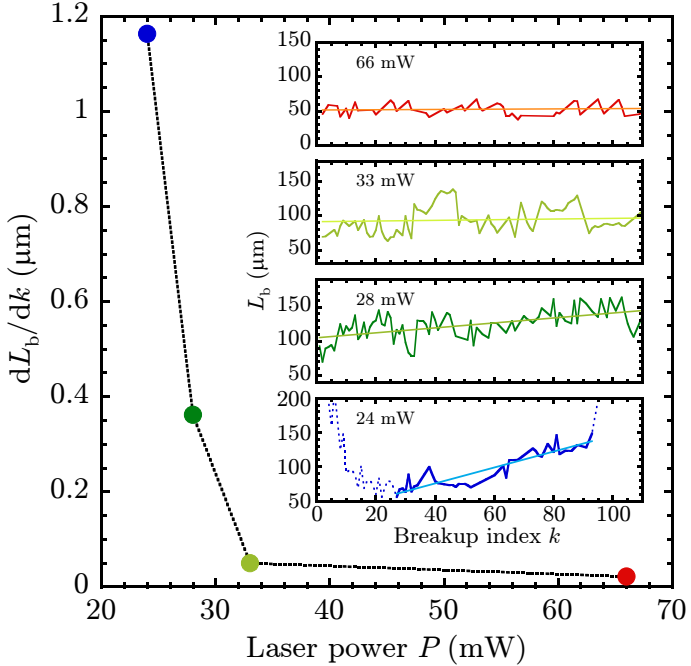

Figure 5. Progression rate of the persistence length over successive breakups, $\mathrm{d} L_{\mathrm{b}} / \mathrm{d} k$, for distinct laser powers $P$ spanning the whole thread-to-droplets transition in the

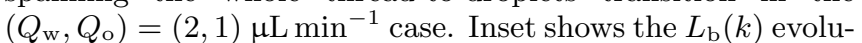
tion in each case, with the corresponding linear fit (restricted to the zone in solid line in the $24-\mathrm{mW}$ case).

taneously reforms.

This investigation thus demonstrates that the thread fragmentation in a confined environment occurs by gradually tuning, via the amplitude of the initial optocapillary pinching, the spatio-temporal development of an instability, from convective to absolute. It also shows the robustness of the mechanisms of the jetting-to-dripping transition in presence of strong confinement.

As a final remark, we note that the linear velocity dependence of the thread-to-droplet forced transition, evidenced by the inset of Fig. 2 (b), also supports this interpretation in terms of a transition from a convective to an absolute instability. In unforced systems, the drippingto-jetting transition indeed occurs at a critical Weber number [45, 48]. It is therefore consistent to find the forced transition shifted towards higher forcing amplitudes when increasing the flow velocity.

\section{WEAKLY-TO-STRONGLY CONFINED TRANSITION}

The forced thread-to-droplet transition in channels is thus unexpectedly smooth, featuring transient regimes as a global instability builds up. Besides, the optocapillary pinching also produces upstream a local bulging [see Fig. 1 (c)] that may partly clog the channel (except gutters in the corners) and thereby prevent or slow down the subsequent breakup. This local confinement should thus produce a rich dynamic behaviour at the single droplet level [35], regardless of the convective or absolute nature of the instability. Figure 6 depicts several temporal sequences that were observed in distinct experimental con-

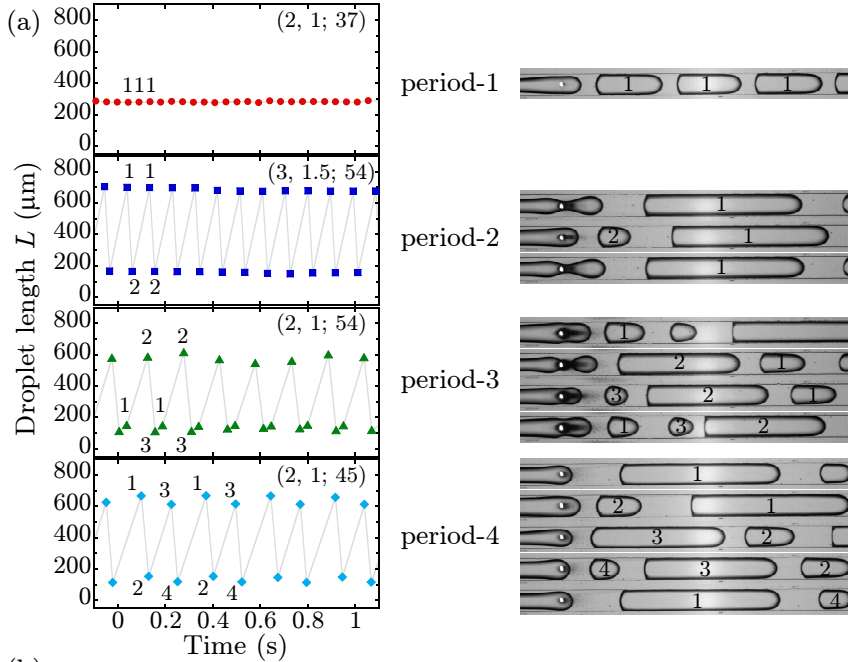

(b)

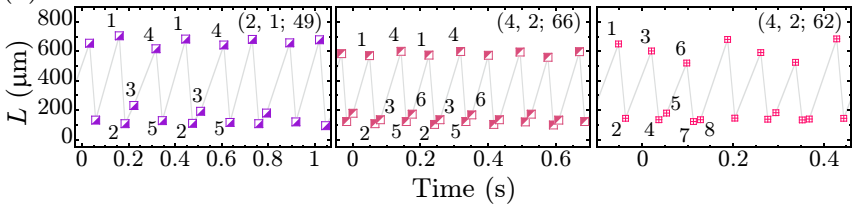

Figure 6. (a) Main fragmentation sequences. From top to bottom: period-1, $-2,-3$ and -4 . (b) Higher-order sequences more sporadically observed; from left to right: period-5, -6 and -8. Experimental conditions are inserted in each graph

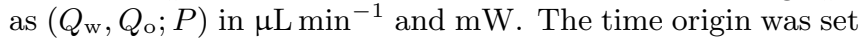
arbitrarily.

ditions. The simplest case, already mentioned above, corresponds to the periodic emission of monodisperse droplets (hereafter referred to as period-1), see Fig. 6 (a), top row. Sequential regimes were also observed, the most common ones are illustrated in Fig. 6 (a):

- The period-2 regime consists of a very long droplet (1) followed by a short one (2);

- The period-3 regime is an alternation between a moderately short (1), a long (2) and a very short droplet (3) — the symmetric $3 \rightarrow 2 \rightarrow 1$ succession was also observed but much more scarcely;

- The period-4 regime corresponds to a couple of period-2 successions entangled (compare droplets 1 and 2 with 3 and 4 , respectively).

To be complete, we also mention three higher-order regimes that were sporadically observed (over a few consecutive periods only), see Fig. 6 (b):

- Period-5: alternation between a period-2 and a period-3 successions;

- Period-6: two distinct period-3 successions entangled (droplet 1 is systematically smaller than 4; droplets 2 and 3 are closer in size than 5 and 6 ); 
- Period-8: alternation between a period-4 and a period-3 immediately followed by a single short droplet.

These sequences were observed reproducibly, over long times, under given experimental conditions. The left panel of Fig. 7 presents the droplet series observed over a few minutes at different laser powers, for $\left(Q_{\mathrm{w}}, Q_{\mathrm{o}}\right)=$ $(2,1) \mu \mathrm{L} \mathrm{min}{ }^{-1}$. The period-1 regime clearly dominates at both low $(37 \mathrm{~mW})$ and high powers $(66 \mathrm{~mW})$, while higher-order sequences (mostly period- 4 at $45 \mathrm{~mW}$ and period-3 at $54 \mathrm{~mW}$ ) are produced in between. Note that several distinct regimes may coexist or alternate over time scales of minutes, as for instance at $41 \mathrm{~mW}$ featuring period-4, period-1 and period-2 regimes successively, in alternation with several occurrences of period-3 sequences. Note also that the period-3 regime is rather scarcely reported in the literature. In a dripping faucet configuration, Subramani et al. predicted a stationary period-3 regime within a small interval in Bond number; small variations beyond this slender window lead either to a monodisperse regime or to intermittent routes to chaos [49]. Here, period-3 sequences are either intermittent (41- and $45-\mathrm{mW}$ cases) or stationary $(54 \mathrm{~mW})$.

The three graphs in the right panel of Fig. 7, where the droplet length is plotted against the laser power, gather the main regimes (those representing less than $1 \%$ of the total population are ignored) obtained for the three investigated flow rates. In all cases, the diagrams feature a similar bifurcation-reconnection shape, though the detailed specific regimes may differ - especially, in the intermediate flow rates case when the period-3 regime is almost absent and appears only intermittently at the opening of the bifurcation. The three diagrams superimpose provided that the laser power is slightly corrected by a factor $\alpha$, close to unity ( $\alpha=1,0.92$ and 0.84 respectively, for increasing flow rates). This remarkably similar trend shows that the bifurcating (period- 1 to period- $n$, with $n \geqslant 2$ ) and reconnecting (period- $n$ to period-1) transitions are primarily driven by the confinement rather than fluid advection. This is particularly noticeable for the largest couple of flow rates $\left(Q_{\mathrm{w}}, Q_{\mathrm{o}}\right)=(4,2) \mu \mathrm{L} \mathrm{min}^{-1}$, in which the low-power period-1 regime and the period1-period-2 bifurcation cannot be triggered due to the fluid advection, yet the droplet formation starts in the same period-2 regime as those observed at lower flow rates. In addition, comparing the experimental images in the low- and high-power period-1 regimes (Fig. 7, cases $\mathrm{i}$ and v; see also Videos 2 and 3 ) actually shows that boundary conditions remain fairly constant during the succession of pinching events: the inflated part of the thread after breakup either never (low power) or always (high power) touches the lateral walls. In contrast, in the period- $n$ regimes these boundary conditions strongly vary from one drop to another: a long droplet forms when the thread touches the wall, and a short one is released when it does not (Fig. 7, cases ii-iv and Video 4). Thus, while this nonlinear behaviour reminds of feedback mechanisms occurring in distinct fluid mechanical systems,

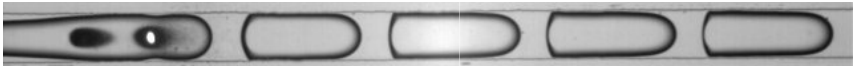

Video 3. Movie illustrating the periodic fragmentation of the thread under continuous laser forcing. The thread interface permanently touches the lateral walls. The real-time dynamics is ten times faster; $P=66 \mathrm{~mW},\left(Q_{\mathrm{w}}, Q_{\mathrm{o}}\right)=$ $(2,1) \mu \mathrm{L} \min ^{-1}$.

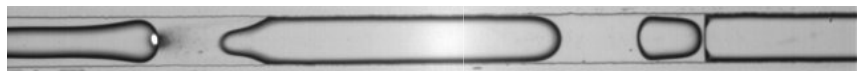

Video 4. Movie illustrating the high-periodic (here, period-4) fragmentation of the thread under continuous laser forcing. The thread interface alternatively does and does not touch the lateral walls. The real-time dynamics is ten times faster; $P=45 \mathrm{~mW},\left(Q_{\mathrm{w}}, Q_{\mathrm{o}}\right)=(2,1) \mu \mathrm{L} \mathrm{min}^{-1}$.

such as the leading edge of a thread in the widely-studied dripping faucet $[45,50]$, or into microfluidic constrictions $[35,51]$, it is here clearly related to the interplay between the pinching and the obstruction due to the channel confinement.

We finally note that, while the droplet length in high periodicity regimes $(n \geqslant 2)$ depend on the laser power, the droplet length is essentially constant in the period1 regime. This length actually coincides well with the wavelength $\lambda^{*}=293 \mu \mathrm{m}$ of the most unstable RP mode calculated according to the linear stability analysis of an unconfined water jet in oil [52]. Retrieving the unconfined most unstable mode in such confined case may sound rather surprising, yet it agrees with the experimental findings of Son et al., who observed that the wavelength converges towards the unconfined one quite abruptly as soon as the confinement between two squeezing walls decreases [53]. This convergence suggests that the RP instability eventually selects the breakup mode although it does not trigger the initial thread pinching.

To summarise, laterally confined threads submitted to a continuous local forcing experience two distinct transitions, that produce non-uniform droplet emission regimes. First, the jetting-to-dripping transition is linked to the establishment of an absolute instability. Second, the weakly-to-strongly confined fragmentation is a signature of the interplay between the imposed deformation and the partial obstruction of the channel near the pinching point. Since they rely on distinct mechanisms, these two transitions are independent and may occur either successively or simultaneously, depending on the thread velocity and the channel geometry, respectively.

\section{CHOPPED FORCING: FROM INSTABILITY-DOMINATED TO CONTROLLED FRAGMENTATION}

Digital microfluidic applications usually require the production of well-defined and calibrated droplets. In 

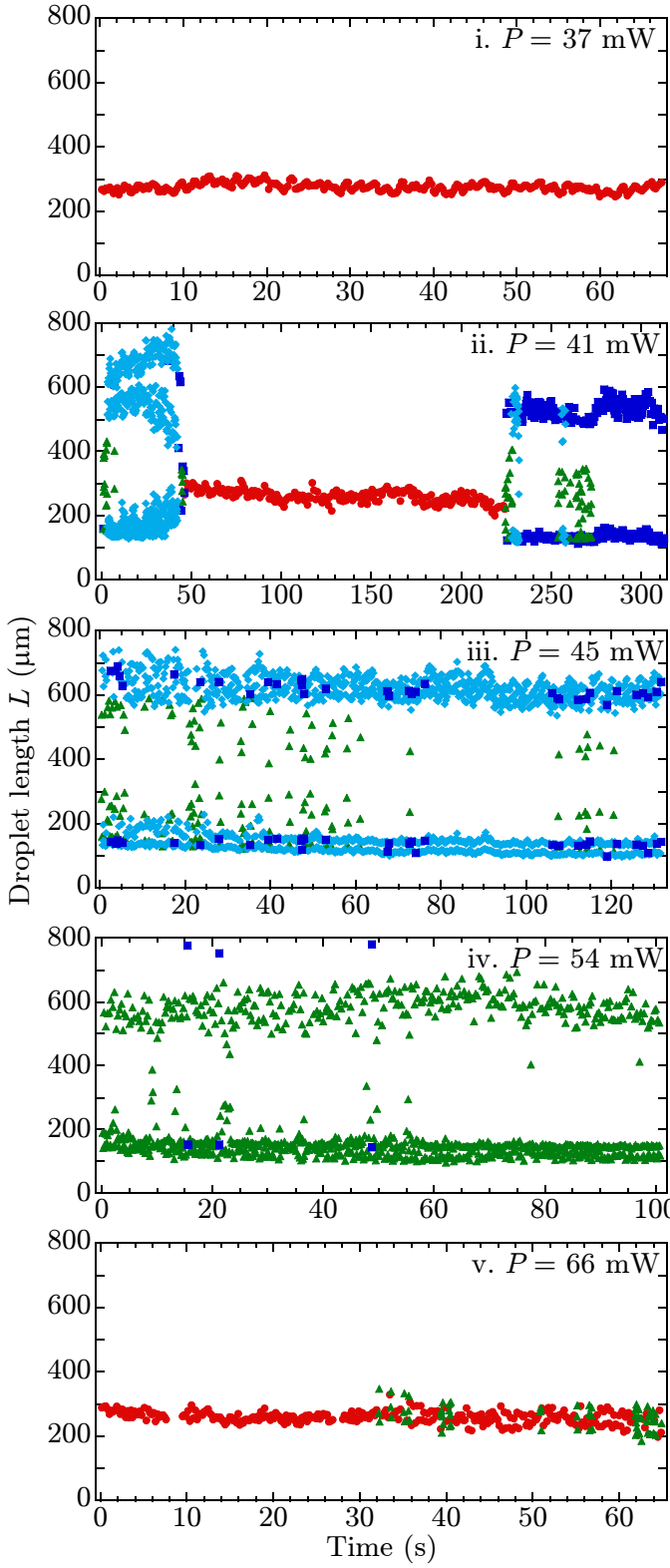
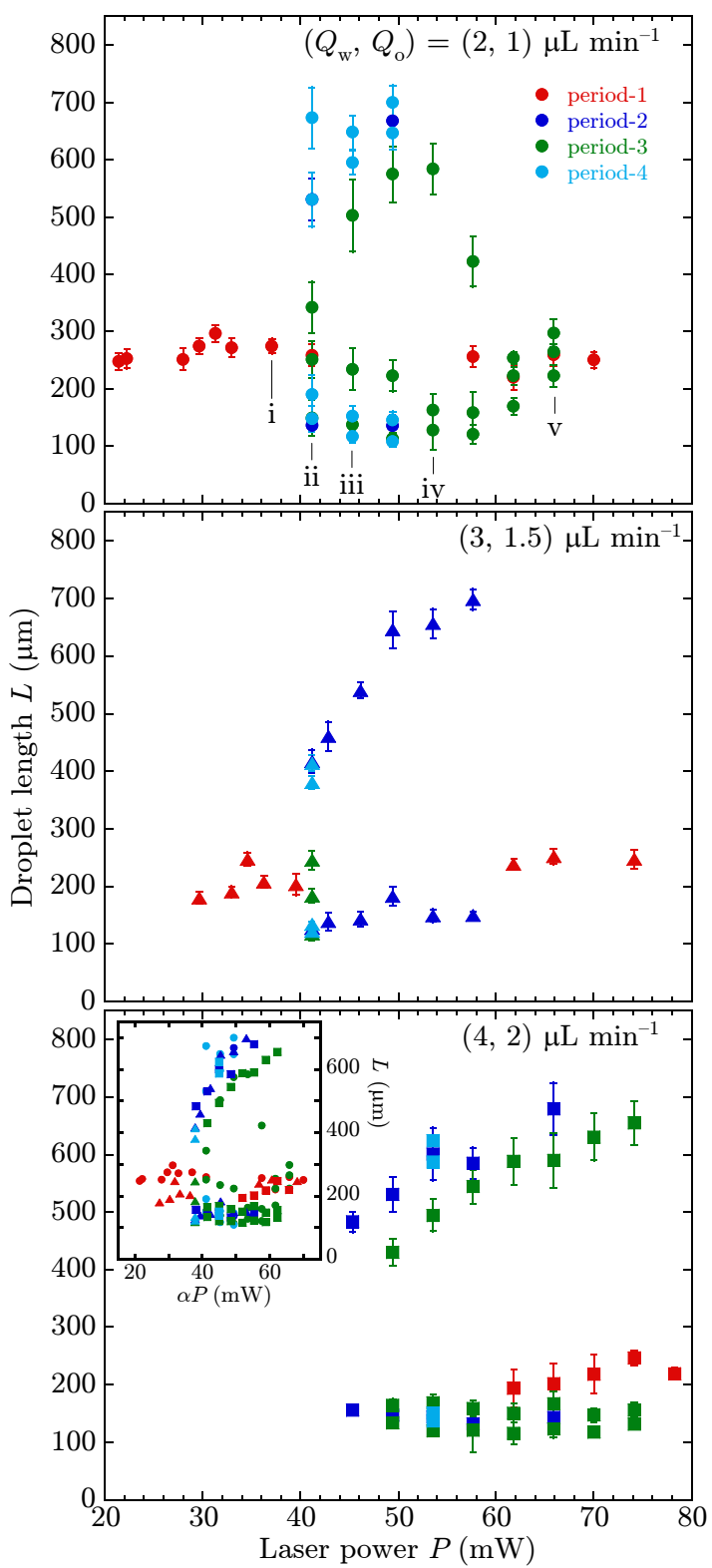

Figure 7. Left: Examples of temporal fragmentation patterns observed when gradually increasing laser power. From top to bottom, $P=37,41,45,54$ and $66 \mathrm{~mW} ;\left(Q_{\mathrm{w}}, Q_{\mathrm{o}}\right)=(2,1) \mu \mathrm{L} \mathrm{min}{ }^{-1}$. Right: Bifurcation diagrams representing the main regimes obtained for the three couples of flow rates investigated. Error bars are \pm 1 standard deviation. Inset in the bottom graph: superimposed bifurcation diagrams for the three couples of flow rates through a corrected power $\alpha P$. Circles, triangles and squares correspond to the three couples of flow rates in increasing order.

this scope, avoiding both transient and inherent highperiodicity fragmented regimes is crucial. Besides, a continuous laser forcing does not allow to select a specific droplet size in the presence of confinement. We thus modulate the laser forcing at the frequency $f$, by inserting a mechanical chopper (SRS SR 540) in the optical path, which produces step-shaped light pulses of duration $1 /(2 f)$ alternating with 'dark' half periods (no signal). Contrary to conventional modulation, which usually produces a sinusoidal signal and therefore only excites a well defined sine mode, here the excitation is continuous-like during the pulse. All modes of wavelength shorter than the slice of liquid flowing during the pulse (first half-period) get excited. The chopper frequency thus defines the upper limit of the excited wavelengths, $\lambda_{\max }=v_{\|} /(2 f)$, with $v_{\|}$the interface velocity.

\section{A. Phase diagrams}

Figure 8 depicts the thread-to-droplet transition in the form of a 'phase diagram' in the (frequency, power) 

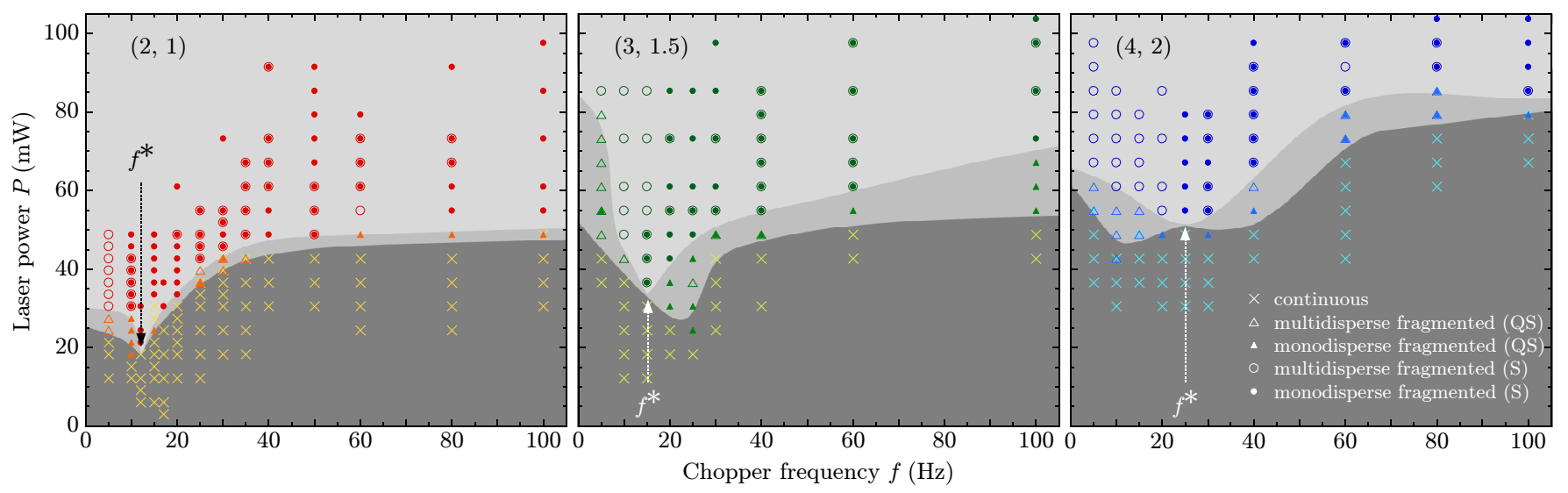

Figure 8. 'Phase diagrams' in the $(f, P)$ plane for the three couples of flow rates $\left(Q_{\mathrm{w}}, Q_{\mathrm{o}}\right)$ investigated. Dark and light grey zones approximately delimit the continuous and steady fragmented states, respectively, medium grey depicts the coexistence region (quasi-stationary fragmented and continuous alternating). Open and filled symbols denote multi- and monodisperse droplet regimes, respectively. The flow rates are indicated in the top left corner in $\mu \mathrm{L} \mathrm{min}{ }^{-1} ; f^{*}$ is the critical forcing frequency corresponding to the direct transition from the continuous to the fragmented regime.

plane, for the three couples of flow rates investigated. In analogy with the continuous forcing case, we identify three regimes: 'continuous' [crosses, almost no fragmentation, including situations analogous to Fig. 2 (a)-top], quasi-stationary 'fragmented (QS)' [triangles, analogous to Fig. 2 (a)-middle], and stationary 'fragmented (S)', [circles, analogous to Fig. 2 (a)-bottom]. These regimes approximately lie within the dark, medium and light grey areas, respectively.

The general shape of the three diagrams is remarkably similar, the transitions between thread and droplets regimes being shifted roughly linearly in beam power when increasing the flow rates linearly. Moreover, considering the two transitions between the three regimes together allows to identify a critical forcing frequency, $f^{*}$ (dotted arrows), that favours the direct transition from the continuous to the stationary fragmented regimes without crossing the intermediate convective-to-absolute transition region. This frequency also seems to increase linearly with the flow rates, suggesting that the excitation is somehow synchronised with a preferred mode of the thread instability. The maximal wavelength excited for each chopper frequency is calculated from the interface velocity, measured for the three couples of flow rates investigated according to the procedure detailed in Appendix A. The phase diagrams are then replotted together in the $\left(\lambda_{\max }, \tau_{\text {adv }} / \tau_{\text {opt }}\right)$ space in Fig. 9 . The regimes corresponding to the three couples of flow rates overlap very well. Moreover, as already illustrated in the continuous optical forcing case, we retrieve the fact that the thread-to-droplet transition occurs for $\tau_{\text {adv }} / \tau_{\text {opt }}$ in the order of 1 . Finally, the direct continuous-steady fragmented transition is found near $\lambda_{\max } \simeq 315 \mu \mathrm{m}$, a value very close to the most unstable mode of the RP instability $\lambda^{*}=293 \mu \mathrm{m}$ - equivalently, the chopper frequency associated to this mode is $f^{*}=v_{\|} /\left(2 \lambda^{*}\right)=13.0$, 19.8 and $24.9 \mathrm{~Hz}$ for the three couples of flow rates, in very good agreement with the values observed in Fig. 8 ( $12 \pm 3,15 \pm 5$ and $25 \pm 5 \mathrm{~Hz}$, respectively). The thread-todroplet transition is also remarkably asymmetric around this value, evidencing two distinct breakup regimes. Below $\lambda^{*}$, the thread fragmentation requires more energy as $\lambda_{\max }$ decreases, while it remains relatively constant above $\lambda^{*}$. This behaviour confirms that the most unstable mode $\lambda^{*}$ dominates the breakup process provided that it is excited (i.e., when $\lambda_{\max } \geqslant \lambda^{*}$ ); shorter wavelengths correspond to slower modes which require a stronger solicitation. In other words, the forced fragmentation requires that the full deformation and destabilisation steps (the optocapillary pinching and the RP instability) proceed while the neck remains within the unstable zone defined in section III B, which is facilitated when fast RP modes are excited. The thread-to-droplet transition depicted in this 'phase diagram' is thus a signature of the dispersion relation of the confined thread.

In addition, even short wavelength solicitations can still lead to the breakup of the thread. This appears in contradiction with the Plateau criterion for stability of a liquid cylinder, which states that only the modes longer than the thread perimeter (here, $\lambda_{0}=173 \mu \mathrm{m}$, corresponding to $f_{0}=22.0,33.6$ and $42.3 \mathrm{~Hz}$ for the three couples of flow rates) are unstable [3]. In fact, the optocapillary forcing intrinsically pinches the thread and, if sufficiently strong, may force the breakup by itself due to the strong pushing flow induced around the neck, regardless of the RP instability [34]. This 'scissorscut' forcing mechanism thus contrasts with remote forcing approaches using pressure transducers [25, 26], actuation channels [54], or previously-reported low-power laser heating [28], that modulate the pressure of an already unstable thread to excite a specific mode but do not exert any local action that could force the breakup of an initially stable thread.

Thus, depending on the excitation and stability con- 


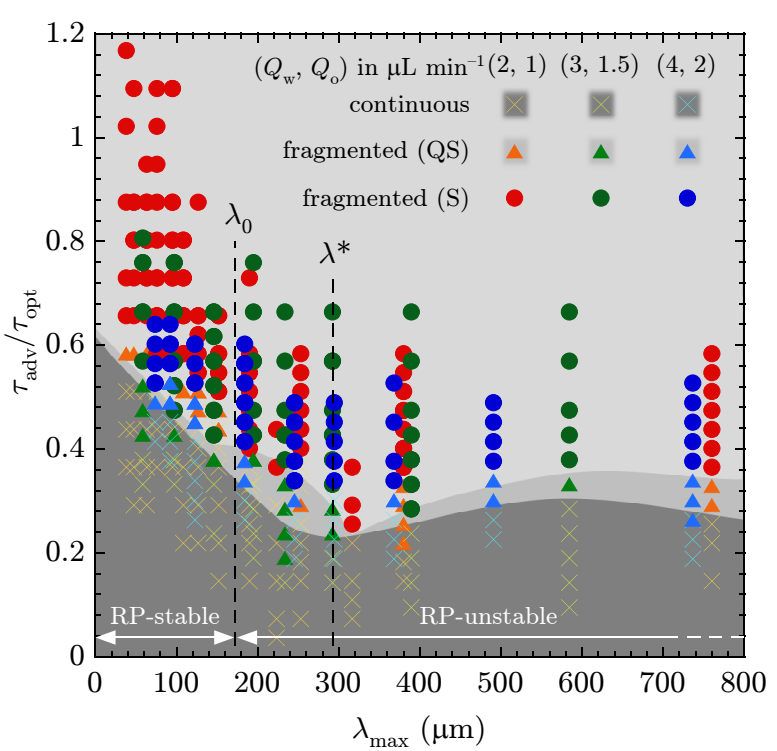

Figure 9. 'Phase diagrams' of Fig. 8 replotted in the $\left(\lambda_{\max }, \tau_{\text {adv }} / \tau_{\text {opt }}\right)$ space. Multi- and monodisperse droplet regimes are merged. The grey areas approximately delimit the three regimes. When $\lambda_{\max }<\lambda_{0}$, no unstable RP mode is excited and the breakup occurs by direct optocapillary pinching.

ditions, the fragmentation is not necessarily driven by the RP instability, which would essentially select a preferred droplet size according to the fluid properties. Here, mono- and multidisperse droplets are actually produced, as represented in Fig. 8 (mono- and multidisperse regimes denoted by filled and open symbols, respectively). In the low-frequency regime $\left(f<f^{*}\right.$ or equivalently $\lambda_{\max }>\lambda^{*}$ ), several droplets can be emitted during a single pulse, as seen for example in Video 5. The multi-sized droplet regime thus corresponds to a situation when the exposure time by pulse is not commensurate with the time needed to produce a droplet at the same power under constant exposure. Note, in addition, that the pinching-obstruction behaviour occurring during the pulse is similar to that observed in continuous period- $n$ regimes (section IV). Conversely, at high frequencies $\left(f>f^{*}, \lambda_{\max }<\lambda^{*}\right.$, and more importantly beyond $f_{0}$ ), several periods may be necessary to break the thread. The number of required periods depends on the laser power, and may either be constant (for example, one droplet produced every 2 periods, see Video 6 ), or alternate from one droplet to another (for example, 2 periods to produce a droplet, then 3 periods to produce the next droplet, and back to 2 periods, Video 7 ), leading to high-frequency multidisperse regimes. Both mono- and multidisperse regimes can actually alternate over the full acquisition time, as illustrated in Fig. 10. Monodisperse droplets are first produced every period (purple circles), then a bidisperse regime results from the alternation of droplets produced in one and two periods (grey squares), see left inset in Fig. 10, before retriev-
Video 5. Movie illustrating the low-frequency $\left(f<f^{*}\right)$ multidisperse fragmentation of the thread under chopped laser forcing. The real-time dynamics is ten times faster; $P=37 \mathrm{~mW}$, $f=5 \mathrm{~Hz},\left(Q_{\mathrm{w}}, Q_{\mathrm{o}}\right)=(2,1) \mu \mathrm{L} \min ^{-1}$.

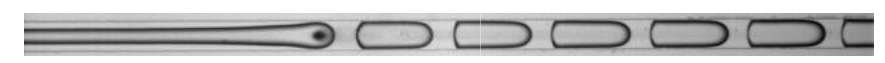

Video 6. Movie illustrating the monodisperse fragmentation (1 drop every 2 periods) of the thread under chopped laser forcing. The real-time dynamics is twenty times faster; $P=$ $67 \mathrm{~mW}, f=50 \mathrm{~Hz},\left(Q_{\mathrm{w}}, Q_{\mathrm{o}}\right)=(2,1) \mu \mathrm{L} \mathrm{min}^{-1}$.

ing the initial one-pulse monodisperse regime. Note that the one-pulse droplets obtained in alternation with twopulse ones are systematically larger than those of the monodisperse sequence, which contrasts with the period1-period-2 transition in continuous forcing cases (see, for instance, case ii in Fig. 7, left).

\section{B. Controlled fragmentation}

Obtaining monodisperse one-pulse droplets requires a sufficient optocapillary forcing to induce breakup within a single half-period. On the one hand, increasing the laser power at high frequency $\left(f>f^{*}\right)$ favours this regime by reducing the number of pulses required to produce one droplet. On the other hand, when this regime is reached, the size of the obtained droplets does not depend anymore on the laser power (Fig. 10, right inset) and only varies with the chopper frequency and flow rates. Consequently, for all frequencies and flow rates, a set of laser powers exists that allows the formation of monodisperse droplets, one per pulse. It is thus possible to control the size of droplets through the chopper frequency. Mass conservation between two consecutive breakups indeed leads to

$$
L=\frac{Q_{\mathrm{w}}}{w h f}+L_{\mathrm{c}}
$$

where the length $L_{\mathrm{c}}$ accounts for the front and rear curvatures, corresponding to the minimal breakup length as discussed in section III B, and is therefore expected close to $w$. The mean thread velocity is calculated according to the full channel cross section, wh, rather than the initial thread cross section $\pi R_{0}^{2} \simeq \pi h^{2} / 4$ since, when droplets

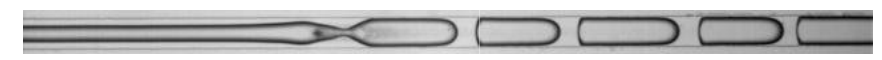

Video 7. Movie illustrating the high-frequency $\left(f>f^{*}\right)$ multidisperse fragmentation of the thread under chopped laser forcing ( 1 drop in 2 and 3 periods in alternation). The realtime dynamics is twenty times faster; $P=55 \mathrm{~mW}, f=50 \mathrm{~Hz}$,

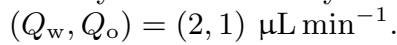




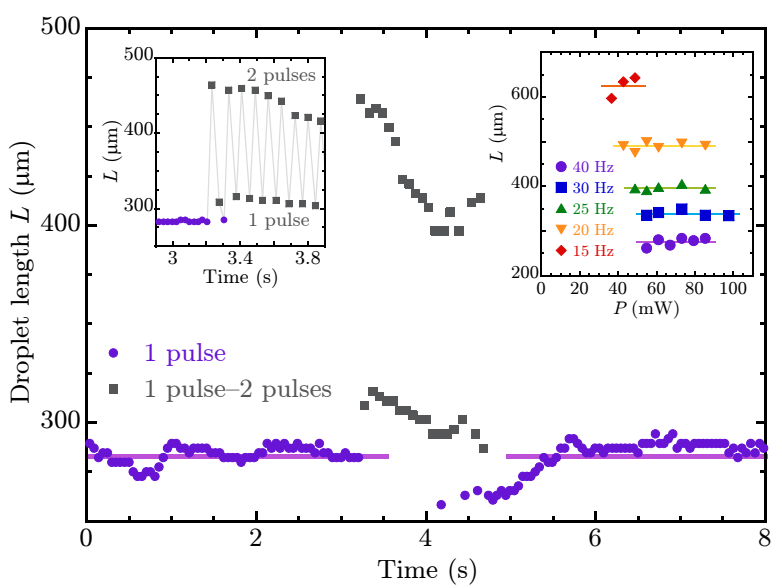

Figure 10. Temporal fragmentation pattern for $P=85 \mathrm{~mW}$ and $f=40 \mathrm{~Hz}$, featuring an alternation of period- 1 (one pulse per droplet, purple circles) and period-2 regimes (one and two pulses per droplet in alternation, grey squares). Left inset: Detailed view of the period-1-period-2 transition. Right inset: Droplet length as a function of the laser power for various chopper frequencies, in stationary or quasi-stationary period1 regimes (one pulse per droplet). The horizontal lines depict the average value for each series. Flow rates are $\left(Q_{\mathrm{w}}, Q_{\mathrm{o}}\right)=$ $(3,1.5) \mu \mathrm{L} \min ^{-1}$.

are produced, the thread locally enlarges up to the lateral walls. The droplet length produced by modulated forcing, in cases when one droplet is released per period, is plotted against the chopper frequency in Fig. 11. Droplets of length comprised between 200 and $600 \mu \mathrm{m}$ are obtained in the frequency range investigated (essentially, $f \geqslant f^{*}$, though values slightly below $f^{*}$ are also taken into account). The trend expected according to Eq. 5 (solid lines) is well retrieved, with values of $L_{\mathrm{c}}$ found between 60 and $77 \mu \mathrm{m}$, reasonably close to the expected value considering the distorted shape of the rear interface. The velocities $v=Q_{\mathrm{w}} /(w h)$ issued from the fit are respectively $5.6,8.5$ and $11.3 \mathrm{~mm} \mathrm{~s}^{-1}$ for increasing flow rate couples. They are $7 \%$ below the expected values (respectively, 6.1, 9.1 and $12.1 \mathrm{mms}^{-1}$ ); this small quantitative disagreement likely results from the crudeness of the model. Nonetheless, they nicely preserve the flow rate ratio as also confirmed by comparing the time to flow a slice of liquid of width $L-L_{\mathrm{c}}$ with $1 / f$ (Fig. 11, inset).

Note finally that droplets produced at $f=f^{*}$ are about $530 \mu \mathrm{m}$ in length, which is very close to the sum of two wavelengths of the most unstable RP mode $\left(2 \lambda^{*}=586 \mu \mathrm{m}\right)$. At this particular frequency, the laser excites the most unstable mode during the full exposure time, which drives the breakup dynamics. The locally inflated thread then advects by $\lambda^{*}$ during the dark half period. When the next pinching starts, the thread has practically retrieved its initial radius $R_{0}$, rather than a larger one due to the upstream bulging induced by the previous pinch-off (see Video 8). This is the reason why this chopper frequency favours a direct thread-to-droplet

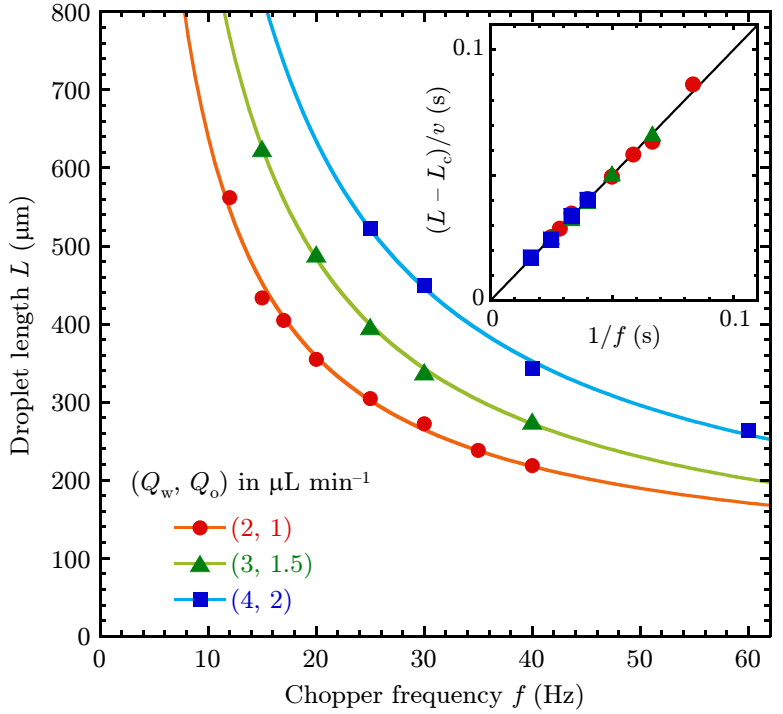

Figure 11. Evolution of the droplet length with chopper frequency in the monodisperse regime, for the three couples of flow rates investigated. Solid line are fits following Eq. 5. Inset: Same data, in reduced scale; the solid line is $y=x$ identity.

Video 8. Movie illustrating the monodisperse fragmentation of the thread under synchronised chopped laser forcing $(f \simeq$ $\left.f^{*}\right)$. The real-time dynamics is ten times faster; $P=31 \mathrm{~mW}$, $f=12 \mathrm{~Hz},\left(Q_{\mathrm{w}}, Q_{\mathrm{o}}\right)=(2,1) \mu \mathrm{L} \min ^{-1}$.

transition, and less power (typically reduced by about $30 \%$ ) is needed to reach the stationary fragmented regime compared to the continuous forcing case.

Chopped forcing thus allows to produce well-calibrated droplets through controlled thread fragmentation. This fragmentation occurs by destabilising an initially stable thread, either by triggering the RP instability, or as a direct result of a strong pinching. The wide operating range of the method, actually limited by the channel width to investigate the confinement effects, illustrates its potential for applications in digital microfluidics.

\section{CONCLUDING REMARKS}

Building a tunable wall-free constriction, we have explored the rich topology patterns featured by a confined thread when locally pinched. First, the thread can successively break and reform, though subject to a continuous and constant forcing. The origin of this breakupreformation alternation is likely connected to the establishment of a convective instability, competing with the natural stability of the thread due to the geometric confinement by the channel $[16,29,30]$. Permanent fragmentation is finally achieved when the triggered instability becomes absolute. Second, the fragmentation can either 
be periodic or follow multidisperse regimes, featuring a remarkable bifurcation-reconnection diagram reminiscent of distinct non-linear fluidic systems [35, 45, 50, 51]. The period-1-period- $n$-period-1 transition actually results from the interplay between the local forcing of the thread and the local obstruction due to the channel confinement. Temporally modulating the optocapillary forcing allows to avoid these period- $n$ regimes, and to favour stationary fragmentation, by synchronising the excitation with the advection of the bulge. It also evidences small wavelength fragmentation of threads, stable against the Plateau criterion [3], by direct optocapillary pinching at high modulation frequencies. In this regime, control of the droplet size is thus achievable through the modulation frequency, provided that the forcing amplitude is sufficient to impose the thread breakup at every shot.

Finally, practically and from a thread actuation viewpoint, externally forcing the thread-to-droplet transition also allows the reversible production of calibrated droplets within confined channels by simply setting or stopping the laser on demand. This reversibility in time is a definite asset compared to passive, geometry-based droplet production devices subject to long-lasting transient stages [42]. In addition, optically-based approaches such as presented here may be displaced on demand, featuring by this way an extended versatility compared to alternatives based on integrated electrical [24] or micromechanical [25] devices, which set actuation in space.

\section{ACKNOWLEDGMENTS}

The authors wish to thank Alexandre Schmit, Laurent Courbin, Pascal Panizza and Hamza Chraïbi for fruitful discussions, and Hamid Kellay for kindly proofreading the manuscript. Valuable experimental help from Sébastien Cassagnère and Joël Plantard is also acknowledged. This work was partly supported by the CNRS and the Conseil Régional d'Aquitaine through grant number 20061102030. The authors would also like to acknowledge networking support by the COST Action MP1205 (http://costmp1205.eu).

\section{Appendix A: Velocity of the interface}

The geometry considered here (a cylindrical thread in a rectangular channel) does not allow the analytical calculation of the velocity profile. We therefore deduced the interface velocity from measurements of the pinching and relaxation dynamics in the wavy regime under chopped optocapillary forcing. These dynamics were taken with a $\times 20$ microscope objective (Olympus SLMPlan, N.A. 0.35 ), the camera operating at 3,000 fps. The laser was modulated at $30 \mathrm{~Hz}$ with a mechanical chopper which produces step-shaped light pulses of duration $1 / 60 \mathrm{~s}$ alternating with $1 / 60 \mathrm{~s}$ dark, and set at various power in

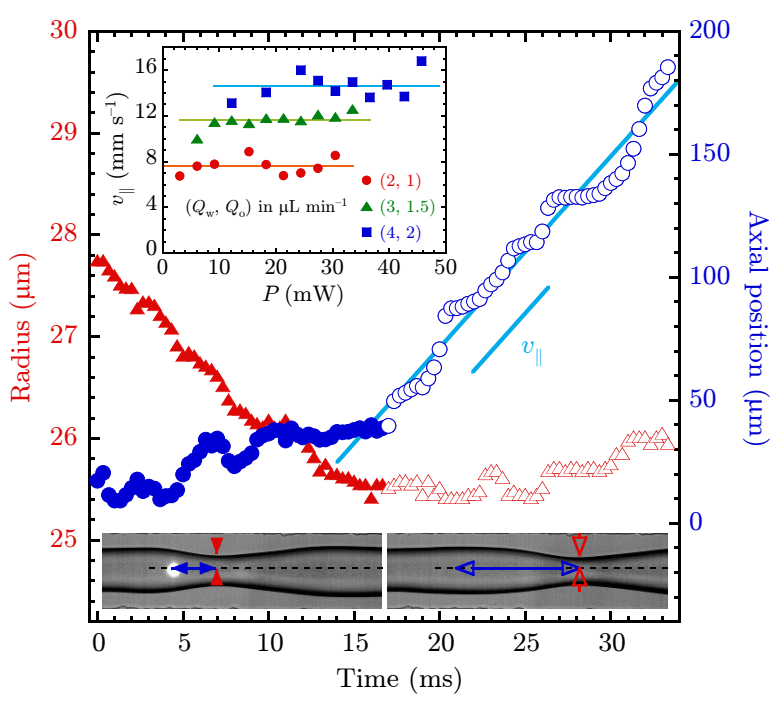

Figure 12. Temporal evolution of the neck radius (red triangles, left scale) and axial position (blue circles, right scale) under moderate pulsed optocapillary forcing, during the halfperiod pulse exposure (filled symbols) and the subsequent relaxation (open symbols) during the following dark half-period. The solid line is a linear fit of slope $v_{\|}$. The filled (resp. open) red and blue arrows on the bottom left (resp. right) images set the definition of the neck radius and its axial position. Flow rates and laser power are $(2,1) \mu \mathrm{Lmin}^{-1}$ and $9 \mathrm{~mW}$, respectively. Inset: Interfacial velocities $v_{\|}$measured during the relaxation stage plotted against laser power for three couples of flow rates. The horizontal lines depict the average value for each series.

the wavy regime. Each measurement consists of the average of ten successive pinching-relaxations series.

During one period, the thread pinches under light exposure, then slowly relaxes when the laser is off, as depicted in Fig. 12 (red triangles, left scale). The axial position of the neck (blue circles, right scale in Fig. 12) increases linearly, but at a rate systematically smaller when the laser is on (filled symbols) than when off (open symbols). This difference is due to the fact that the pinching concerns distinct fluid elements as it proceeds, as a result the neck (defined as the thinnest part of the thread) advects slower than the interface during the exposure time. Conversely, during the 'dark' half-period the neck approximately follows the interface propagation, though a small correction could nonetheless result from the relaxation dynamics. We thus measure the interface velocity, $v_{\|}$, during this second half-period. Measurements performed at different laser powers are depicted, for each couple of flow rates, in the inset of Fig. 12. The interface velocity is found constant over the power range investigated, confirming that the relaxation of the pinching deformation does not influence the interface advection significantly. More importantly, the average values preserve the flow rate ratio, yielding $v_{\|}=7.60 \pm 0.74,11.63 \pm 0.67$, and $14.62 \pm 1.13 \mathrm{~mm} \mathrm{~s}^{-1}$ for $\left(Q_{\mathrm{w}}, Q_{\mathrm{o}}\right)=(2,1),(3,1.5)$ and $(4,2) \mu \mathrm{L} \mathrm{min}{ }^{-1}$, respectively. 


\section{Appendix B: Transient fragmentation dynamics}

In the transiently fragmented regime, the breakup length varies a lot from one breakup event to the other. At low powers, for example $P=24 \mathrm{~mW}$ at $\left(Q_{\mathrm{w}}, Q_{\mathrm{o}}\right)=$ $(2,1) \mu \mathrm{L} \min ^{-1}$, case represented in Fig. $4, L_{\mathrm{b}}(k)$ starts by strongly decreasing from about $300 \mu \mathrm{m}$ (the extent of the unstable zone) to about $50 \mu \mathrm{m}$ (the channel halfwidth, $w / 2)$, as represented in Fig. 4 (24-mW case). Remarkably, minimal breakup lengths are reproducibly found around the breakup event $k \simeq 25-30$, regardless of the laser power and flow rates considered. This robustness strongly suggests that the initial decrease in $L_{\mathrm{b}}$ is related to the number of droplets flowing in the channel. As a matter of fact, the total capacity of the channel downstream of the laser beam is typically $30 \pm 5$ droplets, assuming each droplet is $200-300-\mu m$ long and followed by a $50-\mu \mathrm{m}$ wide slice of oil. While the channel gradually saturates with droplets, its hydrodynamic resistance increases $[31,32]$ due to its partial obstruction which forces the continuous phase to mainly flow through corners [55]. This rapidly increasing resistance slows down the thread velocity, which comparatively accelerates the instability as $\tau_{\text {adv }} / \tau_{\text {opt }} \propto 1 / v_{\|}$locally increases $-L_{\mathrm{b}}$ decreases and approaches the $w / 2$ limit —, making it transiently absolute-like.

At the same time, the slowing down of the velocity at constant flow rates gradually thickens the thread, as observed in Video 1 after the first breakup events. This lateral expansion reinforces the confinement, increasing the thread stability. The breakup location then shifts downstream and the instability turns back convective. Longer droplets are thus produced, increasing the cumulated plug length in the channel, and thereby its hydrodynamic resistance again. Figure 2 (a) top and middle illustrate well such a self-sustained competition. At
$P=21 \mathrm{~mW}$ [Fig. 2 (a), top], the drop size decreases at early stage, then grows until the eventual thread reformation.

In the transiently fragmented regime, optocapillary breakup indeed remains very sensitive to weak random variations in flow and thread radius near the laser beam location and this sensitivity to fluctuations, which drives the first thread breakup, may also make the thread miss a breakup event, leading to its reformation. The reformed thread is transiently thicker than before the first fragmentation event, and takes back its initial cross section after a few seconds - this delay corresponds to the evacuation of the residual droplets out of the channel $\left(v \simeq 5 \mathrm{~mm} \mathrm{~s}^{-1}\right.$, section $\mathrm{VB}$ ), or symmetrically to the initial transient breakup succession when droplets fill the channel.

Increasing the laser power strengthens the optocapillary pinching and triggers thread breakup much sooner [37]. The fragmentation dynamics continues to show the initial variations in drop size but becoming less sensitive to fluctuations, ends up with a quasi-stationary regime before thread reformation [Fig. 2 (a), middle, $P=28 \mathrm{~mW}$. A close view on initial breakup events (Fig. 5) also shows that the first breakup now occurs closer to the laser beam due to a more efficient optocapillary pinching. The instability nonetheless remains convected as illustrated by the weak increase of the breakup length due to the slowly increasing hydrodynamic resistance, evidenced by the slow enlargement of the droplets in the quasi-stationary regime [Fig. 2 (a), middle]. Selfsustained evolution still proceeds and eventually leads to the thread reformation, similarly to the lower power case. Finally, the instability becomes and remains absolute at higher laser power when optocapillary pinching dominates variations in thread radius and hydrodynamic resistance; a stationary fragmented regime is reached typically, when $\tau_{\text {adv }} / \tau_{\text {opt }}>0.4$ [Fig. 2 (b), inset].
[1] Osman A. Basaran, "Small-scale free surface flows with breakup: Drop formation and emerging applications," AIChE J. 48, 1842-1848 (2002).

[2] F. Savart, "Mémoire sur la constitution des veines liquides lancées par des orifices circulaires en mince paroi," Ann. Chim. Phys. 53, 337-398 (1833).

[3] J. Plateau, Statique expérimentale et théorique des liquides soumis aux seules forces moléculaires (GauthierVillars, Paris, 1873).

[4] J. W. Strutt, Lord Rayleigh, "On the instability of jets," Proc. London Math. Soc. s1-10, 4-13 (1878).

[5] J. W. Strutt, Lord Rayleigh, "On the capillary phenomena of jets," Proc. R. Soc. Lond. 29, 71-97 (1879).

[6] J. W. Strutt, Lord Rayleigh, "On the instability of a cylinder of viscous liquid under capillary force," Phil. Mag. 34, 145-154 (1892).

[7] R. Seemann, M. Brinkmann, T. Pfohl, and S. Herminghaus, "Droplet based microfluidics," Rep. Prog. Phys. 75, 016601 (2012).

[8] Alfonso M. Gañán Calvo and José M. Gordillo, "Perfectly monodisperse microbubbling by capillary flow focusing," Phys. Rev. Lett. 87, 274501 (2001).

[9] Todd Thorsen, Richard W. Roberts, Frances H. Arnold, and Stephen R. Quake, "Dynamic pattern formation in a vesicle-generating microfluidic device," Phys. Rev. Lett. 86, 4163-4166 (2001)

[10] Helen Song, Joshua D. Tice, and Rustem F. Ismagilov, "A microfluidic system for controlling reaction networks in time," Angew. Chem. Int. Ed. 42, 768-772 (2003).

[11] Shengqing Xu, Zhihong Nie, Minseok Seo, Patrick Lewis, Eugenia Kumacheva, Howard A. Stone, Piotr Garstecki, Douglas B. Weibel, Irina Gitlin, and George M. Whitesides, "Generation of monodisperse particles by using microfluidics: Control over size, shape, and composition," Angew. Chem. Int. Ed. 44, 724-728 (2005).

[12] G. F. Christopher and S. L. Anna, "Microfluidic methods for generating continuous droplet streams," J. Phys. D: Appl. Phys. 40, R319 (2007).

[13] Shelley L. Anna, Nathalie Bontoux, and Howard A. Sone, "Formation of dispersions using "flow focusing" in 
microchannels," Appl. Phys. Lett. 82, 364-366 (2003).

[14] P. Garstecki, I. Gitlin, W. DiLuzio, G. M. Whitesides, E. Kumacheva, and H. A. Stone, "Formation of monodisperse bubbles in a microfluidic flow-focusing device," Appl. Phys. Lett. 85, 2649-2651 (2004).

[15] Shinji Sugiura, Mitsutoshi Nakajima, Satoshi Iwamoto, and Minoru Seki, "Interfacial tension driven monodispersed droplet formation from microfabricated channel array," Langmuir 17, 5562-5566 (2001).

[16] K. J. Humphry, A. Ajdari, A. Fernández-Nieves, H. A. Stone, and D. A. Weitz, "Suppression of instabilities in multiphase flow by geometric confinement," Phys. Rev. E 79, 056310 (2009).

[17] Michael Hein, Jean-Baptiste Fleury, and Ralf Seemann, "Coexistence of different droplet generating instabilities: new breakup regimes of a liquid filament," Soft Matter 11, 5246-5252 (2015).

[18] Jae-Hoon Choi, Seung-Kon Lee, Jong-Min Lim, SeungMan Yang, and Gi-Ra Yi, "Designed pneumatic valve actuators for controlled droplet breakup and generation," Lab Chip 10, 456-461 (2010).

[19] M. Belloul, J.-F. Bartolo, B. Ziraoui, F. Coldren, V. Taly, and A. I. El Abed, "High-throughput formation and control of monodisperse liquid crystals droplets driven by an alternating current electric field in a microfluidic device," Appl. Phys. Lett. 103, 033112 (2013).

[20] Say Hwa Tan, Florine Maes, Benoît Semin, Jérémy Vrignon, and Jean-Christophe Baret, "The microfluidic jukebox," Sci. Rep. 4, 4787 (2014).

[21] Lothar Schmid and Thomas Franke, "SAW-controlled drop size for flow focusing," Lab Chip 13, 1691-1694 (2013).

[22] Lothar Schmid and Thomas Franke, "Acoustic modulation of droplet size in a T-junction," Appl. Phys. Lett. 104, 133501 (2014).

[23] Zhuang Zhi Chong, Say Hwa Tan, Alfonso M. Gañán Calvo, Shu Beng Tor, Ngiap Hiang Loh, and NamTrung Nguyen, "Active droplet generation in microfluidics," Lab Chip 16, 35-58 (2016).

[24] P. He, H. Kim, D. Luo, M. Marquez, and Z. Cheng, "Low-frequency ac electro-flow-focusing microfluidic emulsification," Appl. Phys. Lett. 96, 174103 (2010).

[25] Iwona Ziemecka, Volkert van Steijn, Ger J. M. Koper, Michel Rosso, Aurelie M. Brizard, Jan H. van Esch, and Michiel T. Kreutzer, "Monodisperse hydrogel microspheres by forced droplet formation in aqueous two-phase systems," Lab Chip 11, 620-624 (2011).

[26] Alban Sauret and Ho Cheung Shum, "Forced generation of simple and double emulsions in all-aqueous systems," Appl. Phys. Lett. 100, 154106 (2012).

[27] Elena Castro-Hernández, Pablo García-Sánchez, Say Hwa Tan, Alfonso M. Gañán Calvo, Jean-Christophe Baret, and Antonio Ramos, "Breakup length of AC electrified jets in a microfluidic flow-focusing junction," Microfluid. Nanofluid. 19, 787-794 (2015).

[28] M. L. Cordero, F. Gallaire, and C. N. Baroud, "Quantitative analysis of the dripping and jetting regimes in coflowing capillary jets," Phys. Fluids 23, 094111 (2011).

[29] M. San Miguel, M. Grant, and J. D. Gunton, "Phase separation in two-dimensional binary fluids," Phys. Rev. A 31, 1001-1005 (1985).

[30] P. Guillot, A. Colin, and A. Ajdari, "Stability of a jet in confined pressure-driven biphasic flows at low Reynolds number in various geometries," Phys. Rev. E 78, 016307
(2008).

[31] Matthew T. Sullivan and Howard A. Stone, "The role of feedback in microfluidic flow-focusing devices," Phil. Trans. R. Soc. A 366, 2131-2143 (2008).

[32] A. Schmit, L. Salkin, L. Courbin, and P. Panizza, "Cooperative breakups induced by drop-to-drop interactions in one-dimensional flows of drops against micro-obstacles," Soft Matter 11, 2454-2460 (2015).

[33] P. Guillot and A. Colin, "Stability of parallel flows in a microchannel after a T junction," Phys. Rev. E 72, 066301 (2005).

[34] Volkert van Steijn, Chris R. Kleijn, and Michiel T. Kreutzer, "Flows around confined bubbles and their importance in triggering pinch-off," Phys. Rev. Lett. 103, 214501 (2009).

[35] P. Garstecki, M. J. Fuerstman, and G. M. Whitesides, "Nonlinear dynamics of a flow-focusing bubble generator: An inverted dripping faucet," Phys. Rev. Lett. 94, 234502 (2005).

[36] P. A. Monkewitz, "The role of absolute and convective instability in predicting the behaviour of fluid systems," Eur. J. Mech. B/Fluids 9, 395-413 (1990).

[37] Matthieu Robert de Saint Vincent, Hamza Chraïbi, and Jean-Pierre Delville, "Optical flow focusing: Lightinduced desabilization of stable liquid threads," Phys. Rev. Applied 4, 044005 (2015).

[38] D. C. Duffy, J. C. McDonald, O. J. A. Schueller, and G. M. Whitesides, "Rapid prototyping of microfluidic systems in poly(dimethylsiloxane)," Anal. Chem. 70, 4974-4984 (1998).

[39] M. Robert de Saint Vincent and J.-P. Delville, "Thermocapillary migration in small-scale temperature gradients: Application to optofluidic drop dispensing," Phys. Rev. E 85, 026310 (2012).

[40] H. Chraïbi and J.-P. Delville, "Thermocapillary flows and interface deformations produced by localized laser heating in confined environment," Phys. Fluids 24, 032102 (2012).

[41] Z. Khattari, P. Steffen, and T. M. Fischer, "Migration of a droplet in a liquid: effect of insoluble surfactants and thermal gradient," J. Phys.: Condens. Matter 14, 4823 (2002).

[42] M. Robert de Saint Vincent, S. Cassagnère, J. Plantard, and J.-P. Delville, "Real-time droplet caliper for digital microfluidics," Microfluid. Nanofluid. 13, 261-271 (2012).

[43] M. Robert de Saint Vincent, J. Petit, M. Aytouna, J.-P. Delville, D. Bonn, and H. Kellay, "Dynamic interfacial tension effects in the rupture of liquid necks," J. Fluid Mech. 692, 499-510 (2012).

[44] B. Dollet, W. van Hoeve, J.-P. Raven, P. Marmottant, and M. Versluis, "Role of the channel geometry on the bubble pinch-off in flow-focusing devices," Phys. Rev. Lett. 100, 034504 (2008).

[45] C. Clanet and J. C. Lasheras, "Transition from dripping to jetting," J. Fluid Mech. 383, 307-326 (1999).

[46] J. N. Israelachvili, Intermolecular and Surface Forces (Third Edition) (Academic Press, San Diego, 2011).

[47] A. S. Utada, A. Fernandez-Nieves, J. M. Gordillo, and D. A. Weitz, "Absolute instability of a liquid jet in a coflowing stream," Phys. Rev. Lett. 100, 014502 (2008).

[48] Carsten Cramer, Peter Fischer, and Erich J. Windhab, "Drop formation in a co-flowing ambient fluid," Chem. Eng. Sci. 59, 3045-3058 (2004). 
[49] Hariprasad J. Subramani, Hak Koon Yeoh, Ronald Suryo, Qi Xu, Bala Ambravaneswaran, and Osman A. Basaran, "Simplicity and complexity in a dripping faucet," Phys. Fluids 18, 032106 (2006).

[50] B. Ambravaneswaran, S. D. Phillips, and O. A. Basaran, "Theoretical analysis of a dripping faucet," Phys. Rev. Lett. 85, 5332-5335 (2000).

[51] M. Hashimoto and G. M. Whitesides, "Formation of bubbles in a multisection flow-focusing junction," Small 6, 1051-1059 (2010).

[52] S. Tomotika, "On the instability of a cylindrical thread of a viscous liquid surrounded by another viscous fluid,"
Proc. R. Soc. Lond. A 150, 322-337 (1935).

[53] Y. Son, N. S. Martys, J. G. Hagedorn, and K. B. Migler, "Suppression of capillary instability of a polymeric thread via parallel plate confinement," Macromolecules 36, 5825-5833 (2003).

[54] H. Willaime, V. Barbier, L. Kloul, S. Maine, and P. Tabeling, "Arnold tongues in a microfluidic drop emitter," Phys. Rev. Lett. 96, 054501 (2006).

[55] F. P. Bretherton, "The motion of long bubbles in tubes," J. Fluid Mech. 10, 166-188 (1961). 